\title{
CAD Scheme To Detect Brain Tumour In MR Images using Active Contour Models and Tree Classifiers
}

\author{
R.Helen ${ }^{\dagger}$ and N.Kamaraj*
}

\begin{abstract}
Medical imaging is one of the most powerful tools for gaining information about internal organs and tissues. It is a challenging task to develop sophisticated image analysis methods in order to improve the accuracy of diagnosis. The objective of this paper is to develop a Computer Aided Diagnostics (CAD) scheme for Brain Tumour detection from Magnetic Resonance Image (MRI) using active contour models and to investigate with several approaches for improving CAD performances. The problem in clinical medicine is the automatic detection of brain Tumours with maximum accuracy and in less time. This work involves the following steps: i) Segmentation performed by Fuzzy Clustering with Level Set Method (FCMLSM) and performance is compared with snake models based on Balloon force and Gradient Vector Force (GVF), Distance Regularized Level Set Method (DRLSE). ii) Feature extraction done by Shape and Texture based features. iii) Brain Tumour detection performed by various tree classifiers. Based on investigation FCMLSM is well suited segmentation method and Random Forest is the most optimum classifier for this problem. This method gives accuracy of $97 \%$ and with minimum classification error. The time taken to detect Tumour is approximately 2 mins for an examination (30 slices).
\end{abstract}

Keywords: Computer aided diagnostics, Level set method, Segmentation, Feature extraction, Feature selection, Tree classifiers

\section{Introduction}

Medical imaging plays a vital role in detecting and treating virtually all types of Cancer. The key characteristics of imaging in cancer are early detection of Cancer, enable less invasive Cancer diagnosis and treatment and foster more effective management of Cancer. A key problem in medical imaging is automatically segmenting an image into its constituent non homogeneous processes. Automatic segmentation has the ability to positively impact clinical medicine by freeing physicians from the burden of manual labelling and by providing robust, quantitative measurements to aid in diagnosis and disease type. One such problem in clinical medicine is the automatic detection of brain Tumours. This problem is handled by various researchers [1-14] and related summary is given in Table 1.

The aim of this paper is to develop improved CAD scheme to detect Brain tumour with maximum accuracy. Segmentation and Classification plays a vital role in CAD. The field of image segmentation kept continuously developing for almost 40 years, and a number of algorithms came forth steadily every year.

In recent years, active contour models have been increasingly and widely used in image processing. These

$\dagger \quad$ Corresponding Author: Dept. of Electrical and Electronic Engineering, Thiagarajar College of Engineering, Madurai, Tamilnadu, India. (rheee@tce.edu)

* Dept. of Electrical and Electronic Engineering, Thiagarajar College of Engineering, Madurai, Tamilnadu, India. (nkeee@tce.edu) Received: April 3, 2014; Accepted: October 21, 2014 methods all need to initialize a closed curve, also called the evolving curve [15], in the image to be segmented and then evolve it driven by a Partial Differential Equation (PDE) until the evolving curve converges. According to the representation of the evolving curve, active contour models can be classified as explicit [16] and implicit [17] categories. Snake a typical explicit active contour model, uses parametric equations to explicitly represent the evolving curve. Implicit active contour models, i.e., level set methods [18] replace the parametric curve with a signed distance function, i.e., the level set function. A level set function is a real-valued function of multiple variables; when the function takes a constant value, e.g., zero, the obtained set is the zero level set which is used to represent the evolving curve. Thus, the evolving curve is implicitly represented by the zero set of the level set function. This representation results implicit active contour models that could handle the topological changing more conveniently than the explicit active contour models. This paper describes a CAD scheme for Brain Tumour detection using active contour models and investigates several approaches to improve CAD performances. This work involves following steps: i) Segmentation is done based on Fuzzy C-Means Level Set Methods (FCMLSM) and the performance is compared with snake models based on Balloon force and Gradient Vector Force (GVF), Distance Regularized Level Set Method (DRLSE) and. ii) Feature extraction is by Shape based features and Texture features and iii) Brain Tumour detection is performed by various 
Table 1. Summary of various methods in automatic brain tumour detection

\begin{tabular}{|c|c|c|c|c|}
\hline Authors & Description & Cases/Images & Accuracy & Time \\
\hline Phillips et al (1995) [1] & Fuzzy clustering & 1 & N/A & N/A \\
\hline Peck et al(1996) [2] & Eigen image analysis & 10 & $\mathrm{~N} / \mathrm{A}$ & $\mathrm{N} / \mathrm{A}$ \\
\hline Zhu and Yan (1997) [3] & Hopfield neural network and active contours & 2 & N/A & N/A \\
\hline Clark et al (1998) [4] & Knowledge based fuzzy clustering & 7 & $70 \%$ & N/A \\
\hline Karayiannis \& pin(1999) [5] & Fuzzy clustering & 1 & N/A & N/A \\
\hline Vinitsku et al (1999) [6] & k-Nearest Neighbour & 9 & N/A & $2 \min$ \\
\hline Fletcher-Heath et al (2001)[7] & Knowledge based fuzzy clustering & 6 & $53 \%-90 \%$ & N/A \\
\hline Kaus et al (2001) [8] & Statistical classification with atlas prior & 20 & $99 \%$ & $10 \mathrm{~min}$ \\
\hline Ho et al (2002) [9] & 3D Level sets & 3 & $85 \%-93 \%$ & N/A \\
\hline Prastawa et al (2003) [10] & Statistical classification via EM & 5 & $49 \%-71 \%$ & $100 \mathrm{~min}$ \\
\hline Prastawa et al (2004) [11] & Knowledge - based & 4 & $68 \%-80 \%$ & $90 \mathrm{~min}$ \\
\hline Zhang et al (2004) [12] & Support Vector Machines & 9 & $60 \%-87 \%$ & N/A \\
\hline Lee et al (2005) [13] & Discriminative Random Field and SVM & 7 & $40 \%-89 \%$ & N/A \\
\hline Jason J. Corso et al (2008) [14] & Multilevel Bayesian segmentation & 20 & $27 \%-80 \%$ & $7 \mathrm{~min}$ \\
\hline Proposed method & FCMLSM + Tree Classifiers & 5 & $90 \%-97 \%$ & $2 \mathrm{~min}$ (30 Slices) \\
\hline
\end{tabular}

tree classifiers.

\section{Materials and Methods}

\subsection{Image datasets}

In this work totally 100 clinical MR Brain images of T1, Contrast enhanced T1, and T2 images with CNS tumours are considered for analysis. The images obtained from Lung Image Database Consortium (LIDC)

\subsection{A CAD Scheme}

The proposed method consists of the following steps:

i) Image segmentation using Level Set method

ii) Image post processing for Tumour extraction

iii) Feature extraction

iv) Feature Selection

v) Classification using Tree Classifiers

\subsection{Level Set method}

The level set method was developed in the 1980s by the American mathematicians Stanley Osher and James Sethian. It has become popular in many disciplines. The basic idea of level set method is to represent a contour as the zero level set of a higher dimensional functional cubes as the Level Set Function (LSF) and formulate the motion of the contour as the evolution of the level set function. A desirable advantage of Level Set Method is that they can represent contours of complex topology and are able to handle topological changes, such as splitting and merging, in a natural and efficient way, which is not allowed in parametric active contour models.

\subsubsection{FCMLSM}

Level set methods, which are established on dynamic implicit interfaces and PDEs, have been shown to be effective for medical image segmentation. However to employ those methods, clinical radiologists and even engineering practitioners are often overwhelmed by intensive computational requirements and complex regulation of controlling parameters. This FCMLSM is new fuzzy level set algorithm developed by Bing Nan Li [19] for automated medical image segmentation.

\subsection{Features extraction}

For each pixel in the segmented image, three categories of features are extracted: Shape based features, Texture features based on the spatial gray level (SGLDM) dependence method and Gray level Co-occurrence matrix (GLCM) detail list is given in Table 2.

\subsection{Feature selection}

This section describes the Feature Selection(FS) using SVM and comparing with other feature selection approaches. For this application also SVM based feature selection method is adopted. Using SVM based feature selection 10 features (F7, F8, F5, F6, F14, F11, F3, F12, F1) are

Table 2. List of the features

\begin{tabular}{|c|c|c|c|}
\hline \multirow{2}{*}{$\begin{array}{c}\text { Feature extraction } \\
\text { method }\end{array}$} & \multirow{2}{*}{$\begin{array}{l}\text { No of } \\
\text { features }\end{array}$} & \multicolumn{2}{|c|}{ Feature extracted } \\
\hline & & Feature label & Feature description \\
\hline \multirow{5}{*}{$\begin{array}{c}\text { Shape based } \\
\text { feature } \\
\text { extraction }\end{array}$} & \multirow{5}{*}{5} & F1 & Area \\
\hline & & $\mathrm{F} 2$ & Brightness \\
\hline & & F3 & Compactness \\
\hline & & F4 & Density \\
\hline & & F5 & Irregularity \\
\hline \multirow{5}{*}{ SGLDM } & \multirow{5}{*}{5} & F6 & Intensity mean \\
\hline & & F7 & Intensity variance \\
\hline & & F8 & $\begin{array}{c}\text { Intensity standard } \\
\text { deviation }\end{array}$ \\
\hline & & F9 & Intensity skewness \\
\hline & & F10 & Intensity kurtosis \\
\hline \multirow{4}{*}{ GLCM } & \multirow{4}{*}{4} & F11 & Correlation \\
\hline & & F12 & Energy \\
\hline & & F13 & Contrast \\
\hline & & F14 & Homogeneity \\
\hline
\end{tabular}


selected for classification which reduces computation time without compromising the accuracy.

\subsubsection{SVM based feature selection}

A central problem in machine learning is identifying a representative set of features from which to construct a classification model for a classification task. This work addresses the problem of feature selection for machine learning through a SVM based approach. For this application SVM is very effective for discovering informative features or attributes for classifying the medical images. The worth of a feature is evaluated by using a SVM classifier. Features are ranked by the square of the weight assigned by the SVM. Feature selection for multiclass problems is handled by ranking attributes for each class separately using a one-versus-all method and then "dealing" from the top of each pile to give a final ranking [20]. The total number of features considered are 14 but using SVM based feature selection method best features are selected for classification.

\subsection{Tumour classification}

Amongst other classifiers methods, decision trees have various advantages: Simple to understand and interpret, requires little data preparation, able to handle both numerical and categorical data, possible to validate a model using statistical tests, Robust and performs well with large data in a short time. The classification of tumour is done by following tree classifiers. They are Alternating Decision (AD) Tree, Best-First (BF) Decision Trees(DT), Random Forest (RF), Functional Trees (FT), Decision Stump (DS) and REP Tree.

\section{Performance Evaluation}

To evaluate the performance of the segmentation algorithm, there are many methods available to perform evaluation, In this work region non uniformity and correlation is used.

\subsection{Region non uniformity}

This is a standard method to evaluate performance. This does not require ground-truth information and is defined as

$$
N U=\frac{\left|F_{T}\right|}{\left|F_{T}+B_{T}\right|} * \frac{\sigma_{f}^{2}}{\sigma^{2}}
$$

Where $\sigma^{2}$ represents the variance of the whole image, and $\sigma_{f}^{2}$ represents the foreground variance $F_{T}$ and $B_{T}$ denotes the foreground and background area pixels in the test image It is expected that a well-segmented image will have a non uniformity measure close to 0 , while the worst case corresponds to $\mathrm{NU}=1$.

\subsection{Correlation}

Correlation between two quantities denotes how closely related those two are. A measure of correlation is thus a really good quantity to prove proper segmentation. In this method, a correlation is done between the selected input image and an image formed by superimposing the foreground segmented using the proposed method with the standard background of the input image.

\subsection{Confusion matrix}

To evaluate the performance of the classifier confusion matrix is created. Confusion matrix is a visualization tool typically used in supervised learning. Each column of the matrix represents the instances in a predicted class, while each row represents the instances in an actual class. One benefit of a confusion matrix is that it is easy to see if the system is confusing two classes. Sensitivity is the probability that test result will be positive when the disease is present (true positive rate expressed as percentage). Specificity is the probability that a test result will be negative when the disease is not present (true negative rate expressed as percentage).

\section{Results}

The segmentation is implemented using Matlab 10.0 under windows 7 operating system and classification is implemented by Weka 3.6. All images used in this work are raw clinical MR brain T1 weighted, T2 weighted and contrast enhanced images. The Figs. 1 and 2 shows the

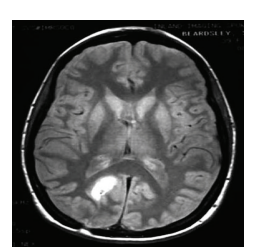

(a)

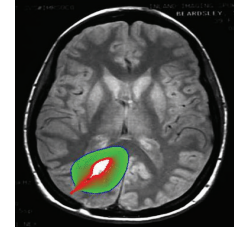

(b)

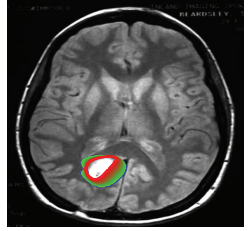

(c)

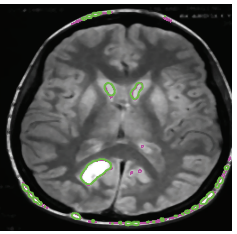

(d)

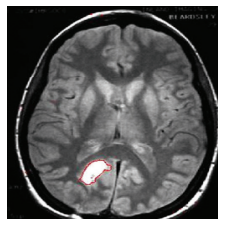

(e)

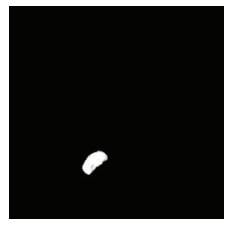

(f)

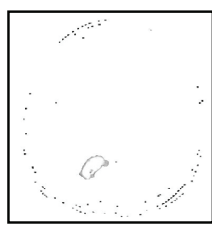

(g)

Fig. 1 (a) MR Brain image (b) - (e) Tumour Segmentation using snake model with balloon force, GVF, Level set and DRLSE, (f) and (g) Extracted tumour using Level set and DRLSE 


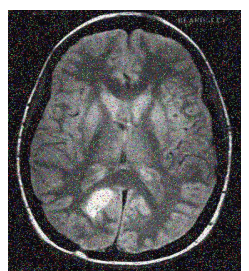

(a)

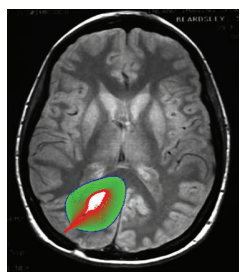

(b)

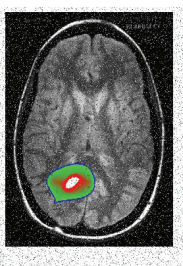

(c)

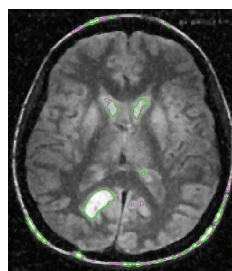

(d)

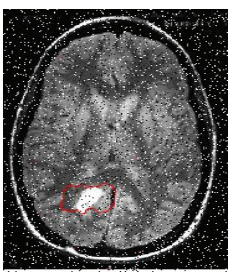

(e)

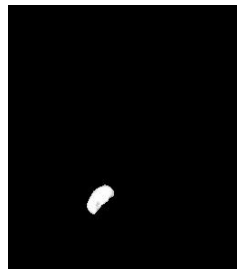

(f)

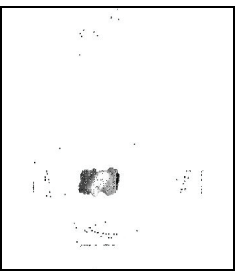

(g)

Fig. 2. (a) MR Brain image with $10 \%$ noise, (b) - (e) Tumor Segmentation using snake model with balloon force, GVF, Level set and DRLSE, (f) and (g) Extracted tumor using Level set and DRLSE

Table 3. Segmentation Performance Evaluations with and without noise

\begin{tabular}{|c|c|c|c|c|c|c|c|c|c|c|c|c|c|c|c|c|}
\hline \multirow{3}{*}{$\begin{array}{c}\text { Examinations } \\
\text { (30 slices / } \\
\text { Exam) }\end{array}$} & \multicolumn{8}{|c|}{ Average Region Non-Uniformity } & \multicolumn{8}{|c|}{ Average Correlation } \\
\hline & \multicolumn{2}{|c|}{ Balloon Force } & \multicolumn{2}{|c|}{ GVF } & \multicolumn{2}{|c|}{ DR LSE } & \multicolumn{2}{|c|}{ FCM LSM } & \multicolumn{2}{|c|}{ Balloon Force } & \multicolumn{2}{|c|}{ GVF } & \multicolumn{2}{|c|}{ DR LSE } & \multicolumn{2}{|c|}{ FCM LSM } \\
\hline & $\begin{array}{l}\text { Withou } \\
\mathrm{t} \text { noise }\end{array}$ & $\begin{array}{c}\text { noise of } \\
10 \%\end{array}$ & $\begin{array}{c}\text { Without } \\
\text { noise }\end{array}$ & $\begin{array}{c}\text { noise of } \\
10 \%\end{array}$ & $\begin{array}{c}\text { Without } \\
\text { noise }\end{array}$ & $\begin{array}{c}\text { noise of } \\
10 \%\end{array}$ & $\begin{array}{c}\text { Without } \\
\text { noise }\end{array}$ & \begin{tabular}{|c|} 
noise \\
of $10 \%$
\end{tabular} & $\begin{array}{c}\text { Without } \\
\text { noise }\end{array}$ & $\begin{array}{c}\text { noise of } \\
10 \%\end{array}$ & $\begin{array}{l}\text { out } \\
\text { se }\end{array}$ & \begin{tabular}{|c|} 
noise of \\
$10 \%$
\end{tabular} & $\begin{array}{c}\text { Without } \\
\text { noise }\end{array}$ & $\begin{array}{c}\text { noise } \\
\text { of } 10 \%\end{array}$ & $\begin{array}{c}\text { Without } \\
\text { noise }\end{array}$ & \begin{tabular}{|c|} 
noise \\
of $10 \%$
\end{tabular} \\
\hline 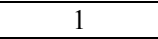 & 0.4631 & 0.5531 & 0.3671 & & & & 0 & 0.01 & & & & & & & 1.00 & 1.00 \\
\hline 2 & 320 & 6231 & 3210 & 21 & 3 & 1 & 0.01 & 0.01 & .4736 & 32 & 13 & 311 & 0.97 & 0.9022 & 0.99 & 0.98 \\
\hline 7 & 421 & 5221 & 0.3107 & 0.4300 & 0.03 & 01 & 0 & 0 & .5681 & 0.4892 & .7090 & 245 & 0.97 & 0.901 & 1.00 & 0.99 \\
\hline$T$ & 4320 & 0.4918 & 0.3110 & 0.3950 & 0.04 & 0.1452 & 0.02 & 0.02 & 0.5775 & 0.5111 & 0.7526 & \begin{tabular}{|l|}
0.6157 \\
\end{tabular} & 0.96 & 0.9521 & 0.98 & 0.98 \\
\hline 5 & 0.4121 & 0.4745 & 0.3120 & 0.3870 & 0.02 & 0.1999 & 0.01 & 0.01 & 0.5960 & 0.5390 & 0.6931 & 0.6214 & 0.98 & 0.9211 & 0.99 & 0.99 \\
\hline
\end{tabular}

Table 4. Classification Results for various classifiers for 10 - fold cross validation

\begin{tabular}{|c|c|c|c|c|c|c|c|c|c|c|c|c|}
\hline \multirow[b]{2}{*}{$\begin{array}{c}\text { Tree } \\
\text { Classifier }\end{array}$} & \multicolumn{2}{|c|}{ Time (sec) } & \multicolumn{2}{|c|}{$\begin{array}{c}\text { Correct } \\
\text { classification \% }\end{array}$} & \multicolumn{2}{|c|}{$\begin{array}{c}\text { Incorrect } \\
\text { classification } \%\end{array}$} & \multicolumn{2}{|c|}{ RMSE } & \multicolumn{2}{|c|}{ Sensitivity $\%$} & \multicolumn{2}{|c|}{ Specificity \% } \\
\hline & $\begin{array}{l}\text { With out } \\
\text { (FS) }\end{array}$ & With (FS) & $\begin{array}{l}\text { With out } \\
\text { (FS) }\end{array}$ & With (FS) & $\begin{array}{l}\text { With out } \\
\text { (FS) }\end{array}$ & With (FS) & $\begin{array}{l}\text { Without } \\
\text { (FS) }\end{array}$ & With (FS) & $\begin{array}{l}\text { With out } \\
\text { (FS) }\end{array}$ & With (FS) & $\begin{array}{l}\text { With out } \\
\text { (FS) }\end{array}$ & With (FS) \\
\hline TApe & 0.02 & 0.02 & 95.6 & 95.6 & 4.4 & 4.4 & 0.18 & 0.184 & 96 & 96.7 & 98 & 81.8 \\
\hline LAD & 0.03 & 0.02 & 93.8 & 95.6 & 6.2 & 4.4 & 0.22 & 0.195 & 96 & 96.7 & 97 & 81.8 \\
\hline FT & 0.03 & 0.03 & 93.8 & 93.8 & 6.2 & 6.2 & 0.22 & 0.225 & 96 & 94.8 & 70 & 75 \\
\hline DS & 0.02 & 0.02 & 96.3 & 96.3 & 3.7 & 3.7 & 0.189 & 0.189 & 97.3 & 97.3 & 83.3 & 83.3 \\
\hline J48 & 0.02 & 0 & 94.4 & 95.1 & 5.6 & 4.9 & 0.229 & 0.215 & 95.4 & 96.1 & 77.8 & 80 \\
\hline $\mathrm{BF}$ & 0.03 & 0.02 & 96.3 & 96.3 & 3.7 & 3.7 & 0.189 & 0.19 & 97.3 & 97.3 & 83.3 & 83.3 \\
\hline NB & 0.17 & 0.13 & 94.4 & 94.4 & 5.6 & 5.6 & 0.225 & 0.225 & 96 & 96 & 72.7 & 72.7 \\
\hline REP & 0.02 & 0.02 & 96.3 & 96.3 & 3.7 & 3.7 & 0.189 & 0.189 & 97.3 & 97.3 & 83.3 & 83.3 \\
\hline RT & 0.02 & 0.02 & 93.8 & 93.8 & 6.2 & 6.2 & 0.248 & 0.248 & 95.4 & 96 & 70 & 66.7 \\
\hline RF & 0.05 & 0.02 & 95.6 & 96.3 & 4.4 & 3.7 & 0.205 & 0.186 & 96.1 & 97.3 & 88.9 & 83.3 \\
\hline
\end{tabular}

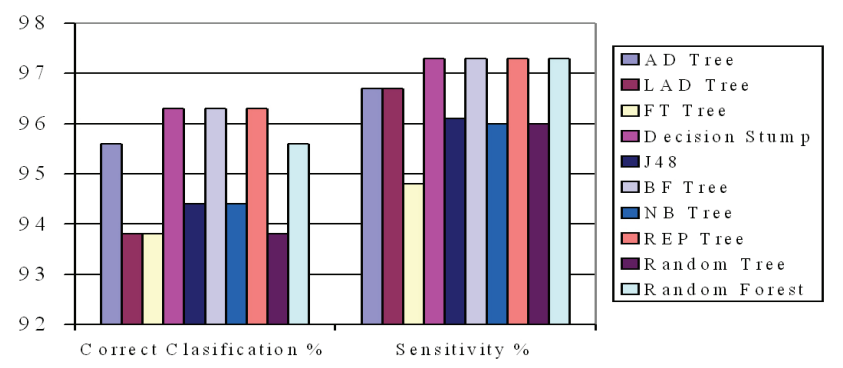

(a)

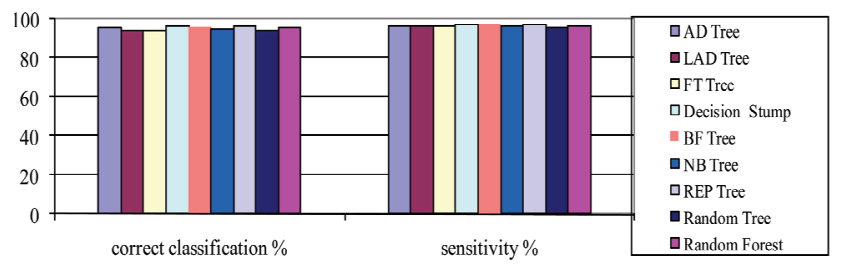

(b)

Fig. 3. Performance Evaluation of classifiers (a) with feature selection (b) without feature selection segmentation result for sample MR Brain images with tumour noiseless and noisy environment respectively. The performance evaluation for segmentation is taken for 5 examinations. The performance Evaluation table for segmentation and classifiers, with and without noise are given in Tables 3 and 4 respectively. The performance evaluation chart is shown in Fig. 3.

\section{Discussions}

The unique characteristic of this work is the investigation and observation a number of important issues in developing and assessing CAD for Brain Tumour detection using our prototype CAD scheme on limited image dataset.

The developed CAD scheme initially deals with Tumour segmentation based on Segmentation performed by Fuzzy Clustering with Level Set Method (FCMLSM) and performance is compared with snake models based on Balloon force and Gradient Vector Force (GVF), Distance 
Regularized Level Set Method (DRLSE). This proposed segmentation method Segmentation based on snake models and DRLSE are to initialize a closed curve so user interaction is needed. But for FCMLSM segmentation user interaction is not needed since initial contour is developed by FCM. Based on experimental results Figs. 1 and 2, Segmentation accuracy of FCMLSM is best when comparing with other techniques. The performance of this method is evaluated by standard techniques for MR brain images with and without noise and the results are tabled in the Table 3 which proves the accuracy of this segmentation method. The detection of abnormalities in MR images which are beyond human appreciation and otherwise difficult to determine by other classical methods of image processing can be detected by using the shape and textural features that has been quantitatively established over the past years. Three categories of features are extracted: Shape based features, Texture features based on the spatial gray level (SGLDM) dependence method and Gray level Co-occurrence matrix (GLCM). From these method a total of 14 features are taken for analysis of MR brain images.

A central problem in machine learning is identifying a representative set of features from which to construct a classification model for a classification task. This work addresses the problem of feature selection for machine learning through a SVM based approach. For this application SVM is very effective for discovering informative features or attributes for classifying the medical images. The worth of a feature is evaluated by using a SVM classifier. Features are ranked by the square of the weight assigned by the SVM. Feature selection for multiclass problems is handled by ranking attributes for each class separately using a one-versus-all method and then "dealing" from the top of each pile to give a final ranking (Xiaoou 1998). The total no. of features considered are 14 but using SVM based feature selection method minimum best features are selected for classification.

Amongst other classifiers methods, decision trees have various advantages: Simple to understand and interpret, requires little data preparation, able to handle both numerical and categorical data, possible to validate a model using statistical tests, Robust and performs well with large data in a short time. From the Table 4, results inferred that the Random Forest and AD Tree classifiers have a correct classification percentage approximately of above $97 \%$ and with minimum classification error. Finally above proposed CAD scheme is suitable for detecting Brain Tumour. In future the method can be enhanced to say the severity of Tumour benign (non-cancerous) or malignant Tumour (cancerous).

\section{Conclusion}

In this paper a CAD scheme is developed to detect Brain Tumour in Magnetic Resonance Brain Images using active contour models and tree classifiers. In this work, 100 clinical MR brain images (T1 weighted and T2 weighted) with Tumour and without Tumour are considered for brain Tumour detection. For segmentation, snake models, DRLSE and FCMLSM are applied. Segmentation based on snake models and DRLSE are to initialize a closed curve so user interaction is needed. But for FCMLSM segmentation user interaction is not needed since initial contour is developed by FCM. Based on experimental results, Segmentation accuracy of FCMLSM is best when comparing with other techniques. For classification, Random Forest and AD Tree classifiers are adopted and give sensitivity of $97 \%$ and with minimum classification error. The time taken to detect Tumour is approximately 2 mins. for an examination(30 slices). By comparing the accuracy and diagnostic time with other method, the proposed method is the best.

\section{References}

[1] Phillips, W.E., Velthuizen, R.P., Phupanich, S., Hall, L.O., Clarke, L.P. and Silbiger, M. L. "Application of fuzzy c-means segmentation technique for tissue differentiation in MR images of a hemorrhagic glioblastoma multiforme," Magnetic Resonance Imaging, Vol. 13, No. 2, pp. 277-290, 1995.

[2] Peck, D.J., Windham, J.P., Emery, L.L., SoltanianZadeh, H. Hearshen, D.O. and Mikkelsen, T. "Cerebral Tumour volume calculations using planimetric and eigen image analysis," Medical Physics, Vol. 23, No. 12, pp. 2035-2042, 1996.

[3] Zhu, Y. and Yan, H. "Computerized Tumour boundary detection using a hopfield neural network," IEEE Transactions on Medical Imaging, Vol. 16, No. 1, pp. 55-67, 1997.

[4] Clark, M.C., Hall, L.O., Goldgof, D.B., Velthuizen, R., Murtagh, R. and Silbiger, M.S. "Automatic Tumour segmentation using knowledge-based techniques," IEEE Transactions on Medical Imaging, Vol. 17, No. 2, pp. 187-201, 1998.

[5] Karayiannis, N. B. and Pai, P.-I. "Segmentation of magnetic resonance images using fuzzy algorithms for learning vector quantization," IEEE Transactions on Medical Imaging, Vol. 18, No. 2, pp. 172-180, 1999.

[6] Vinitski, S., Gonzalez, C. F., Knobler, R., Andrews, D., Iwanaga, T. and Curtis, M. "Fast tissue segmentation based on a 4D feature map in characterization of intracranial lesions," Journal Magnetic Resonance Imaging, Vol. 9, No. 6, pp. 768-776, 1999.

[7] Fletcher-Heath, L.M., Hall, L.O., Goldgof, D.B. and Reed Murtagh, F. "Automatic segmentation of non enhancing brain Tumours in magnetic resonance images," Artificial Intelligent Medicine, Vol. 21, pp. 43-63, 2001.

[8] Kaus, M., Warfield, S., Nabavi, A., Black, P.M., Jolesz, 
F.A. and Kikinis, R. "Automated segmentation of MRI of brain Tumours," Radiology, Vol. 218, pp. 586-591, 2001.

[9] Ho, S., Bullitt, E. and Gerig, G. "Level set evolution with region competition: Automatic 3-D segmentation of brain Tumours," Proceedings of International Conference Pattern Recognition, Vol. 1, pp. 532-535, 2002.

[10] Prastawa, M., Bullitt, E., Bullitt, N., Leemput, K. V. and Gerig, G. "Automatic brain Tumour segmentation by subject specific modification of atlas priors," Academy Radiology, Vol. 10, pp. 1341-1348, 2003.

[11] Prastawa, M., Bullitt, E., Ho, S. and Gerig, G. "A brain Tumour segmentation framework based on outlier detection," IEEE Transactions on Medical Imaging, Vol 8, No. 3, pp. 275-283, 2004.

[12] Zhang, J., Ma, K., Er, M. H. and Chong, V. “Tumour segmentation from magnetic resonance imaging by learning via one-class support vector machine," in Proceedings 2004 International Conference Intelligent Mechatronics Automation, Chengdu, China, pp. 207211, 2004.

[13] Lee, C. H., Schmidt, M., Murtha, A., Bistritz, A., Sander, J. and Greiner, R. "Segmenting brain Tumour with conditional random fields and support vector machines," in Proceedings of Workshop Computer Vision Biomedical Image Application International Conference Computer Vision, pp. 469-478, 2005.

[14] Jason J. Corso, Eitan Sharon, Shishir Dube, Suzie ElSaden, Usha Sinha and Alan Yuille, "Efficient Multilevel Brain Tumour Segmentation With Integrated Bayesian Model Classification", IEEE Transactions on Medical Imaging, Vol. 27, No. 5, 2008.

[15] Osher, S. and Fedkiw, R. "Level Set Methods and Dynamic Implicit Surfaces", New York, SpringerVerlag, ch. 3, 4, 2003.

[16] Kass, M., Witkin, A. and Terzopoulos, D. "Snakes: Active contour models," International Journal Computer Vision, Vol. 1, No. 4, pp. 321-331, 1988.

[17] Li, C., Xu, C., Gui, C. and Fox, M. D. "Level set evolution without re-initialization: A new variational formulation," in Proc. IEEE Conference Computer Vision. Pattern Recognition, Vol. 1, pp. 430-436, 2005.

[18] Malladi, R., Sethian, J. A. and Vemuri, B.C. "Shape modeling with front propagation: A level set approach," IEEE Transactions on Pattern Analysis and Machine Intelligence, Vol. 17, No. 2, pp. 158-175, 1995.

[19] Bing NanLi, CheeKongChui, Stephen Chang and Ong, S.H. "Integrating spatial fuzzy clustering with level set methods for automated medical image segmentation", Journal Computers in Biology and Medicine, Vol. 41, No. 1, pp.1-10, 2011.

[20] Xiaoou Tang, "Texture information in run length matrices", IEEE Transactions on Image Processing, Vol. 7, No. 11, 1998.

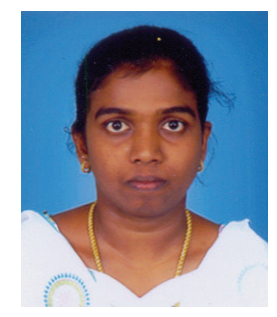

R. Helen obtained Bachelor of Engineer in Instrumentation and Control Engineering at Madras University, Chennai and Master of Engineer in Medical Electronics at Anna University, Chennai. She obtained Ph.D in the medical image segmentation. She has 14 years of experience in teaching. She is currently working as Assistant Professor in Department of Electrical and Electronics Engineering, Thiagarajar college of Engineering, Madurai.

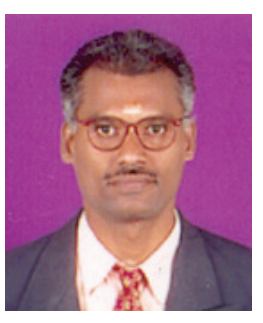

N. Kamaraj is a Professor \& HEAD, Department of Electrical Engineering, Thiagarajar College of Engineering, Madurai, Tamilnadu, India. He has 9 years of Industrial Experience and 26 years of experience in teaching and research. He obtained B.E. degree in Electrical and Electronics Engineering and M.E. degree in Power System Engineering from Madurai Kamaraj University in the year 1988 and 1994 respectively. He obtained Ph.D. Degree in the Power System Security Assessment in the year 2003 from Madurai Kamaraj University. 